\title{
Die traumatische Beugesehnendurchtrennung
}

\author{
Wolfram A. Heitzmann
}

\author{
Beugesehnenverletzungen an der Hand sind ein anspruchsvolles Feld für Chirurg und Thera- \\ peut: Der Operation muss eine differenzierte Diagnostik vorausgehen. Für die Wahl der \\ Nahttechnik gibt es keinen internationalen Standard. Der Heilungsprozess des bradytro- \\ phen Sehnengewebes ist langwierig, und Narbengewebe begünstigt die Bildung von Adhä- \\ sionen und Bewegungseinschränkungen.
}

Nach den Statistiken der gewerblichen Berufsgenossenschaften ist in $40 \%$ der Arbeitsunfälle die Hand betroffen. Somit ist sie die am häufigste betroffene Körperregion. Da sich immer mehr Menschen als Heimwerker versuchen, nimmt außerdem auch die Zahl der Handverletzungen im Freizeitbereich zu. Bei schwereren Handverletzungen, insbesondere wenn Sehnen, Nerven oder Gefäße betroffen sind, ist der Plastische Chirurg bzw. Handchirurg gefordert [1].

Die Prävalenz von Beugesehnenverletzungen ist mit $1 \%$ aller Handverletzungen zwar relativ niedrig, jedoch mit großen sozialen und ökonomischen Folgen verbunden. Wie die klinische Erfahrung zeigt, ist die postoperative Nachsorge und insbesondere die Ergotherapie und Schienenbehandlung nach Beugesehnennaht wesentlich für den Behandlungserfolg [2].

\section{Anatomie der Fingerbeuger}

Die extrinsischen Beugemuskeln der Finger und des Daumens liegen im Unterarm und setzen mit jeweils zwei Sehnen an den Fingern bzw. mit einer Sehne am Daumen an.

Die oberflächliche Beugesehne (Sehne des M. flexor digitorum superficialis, FDS) setzt mit einem ulnaren und einem radialen Seitenzügel an der Mittelgliedbasis an und beugt Grund- und Mittelgelenk. Die tiefe Beugesehne (Sehne des M. flexor digitorum profundus, FDP) tritt in Höhe des proximalen Fingergrundglieds durch die oberflächliche Beugesehne hindurch (sog. Hiatus tendineus) und setzt an der Endgliedbasis an. Sie beugt also alle 3 Fingergelenke. Ab Grundgliedmitte läuft die FDP-Sehne oberflächlich und kann deshalb in diesem Bereich leicht verletzt werden ( $\triangleright$ Abb. 1). Oberflächliche und tiefe Beugesehne sind zudem durch die Vincula longa und breve miteinander verbunden, die aus Überresten eines durchgehenden Mesotendineums aus der Embryonalentwicklung entstehen. Zusätzlich zur Ernährung durch Diffusion aus der Synovia wird die Sehne durch Blutgefäße innerhalb der Vinkula versorgt. Deshalb ist der Erhalt dieser Strukturen für die Sehnenheilung essenziell [3].

Am Daumen verläuft nur eine extrinsische Beugesehne: die Sehne des M. flexor pollicis longus, FPL. Sie ist die einzige Sehne, die den Daumen im Endgelenk beugt. Somit fällt diese Funktion bei ihrer Durchtrennung völlig aus und kann nicht kompensiert werden. Außerdem schwächt eine Durchtrennung der FPL-Sehne die Beugung im Daumengrundgelenk und im Handgelenk ab.

Ring- und Kreuzbänder führen und befestigen die Beugesehnen an den Phalangen. Um ein Bogensehnenphänomen, also das Abheben der Sehne vom Knochen, zu vermeiden, sind die Ringbänder bei Operationen zu schonen [4].

Die intrinsischen Fingerbeuger haben ihren muskulären Ursprung innerhalb der Hand. Hierzu gehören die Mm. lumbricales und Mm. interossei, welche in den Grundgelenken beugen.

\section{Zoneneinteilung}

Die Einteilung in Zonen erlaubt eine bessere Vergleichbarkeit unterschiedlicher Beugesehnenverletzungen ( $\triangleright$ Abb.2). Je nach Zone können unterschiedliche operativ-technische Schwierigkeiten in der Versorgung auftreten.

Zone 1 Läsionen in dieser Zone entsprechen ansatznahen Verletzungen der FDP-Sehne. Sobald distal mehr als $1 \mathrm{~cm}$ Sehnenstumpf für die Naht zur Verfügung steht, kann eine direkte Sehnennaht durchgeführt werden. Ist 


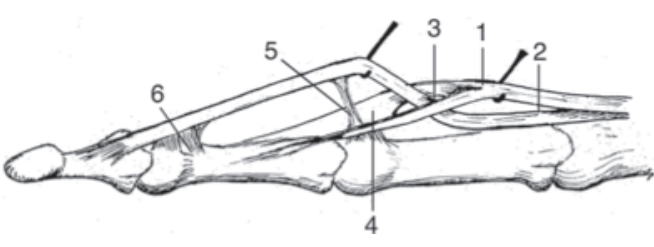

- Abb. 1 Die tiefe Beugesehne tritt auf Höhe der Grundphalanx durch die beiden Zügel der oberflächlichen Beugesehne hindurch und verläuft dann an der Oberfläche. (1) FDS-Sehne, (2) FDP-Sehne, (3) Hiatus tendineus, (4) Chiasma tendineum, (5) Vincula longa, (6) Vincula breve. (Quelle: Hoffmann R, Krimmer H. Anatomie. In: Hoffmann R, Hrsg. Checkliste Handchirurgie. 4. Aufl. Stuttgart: Thieme; 2016)

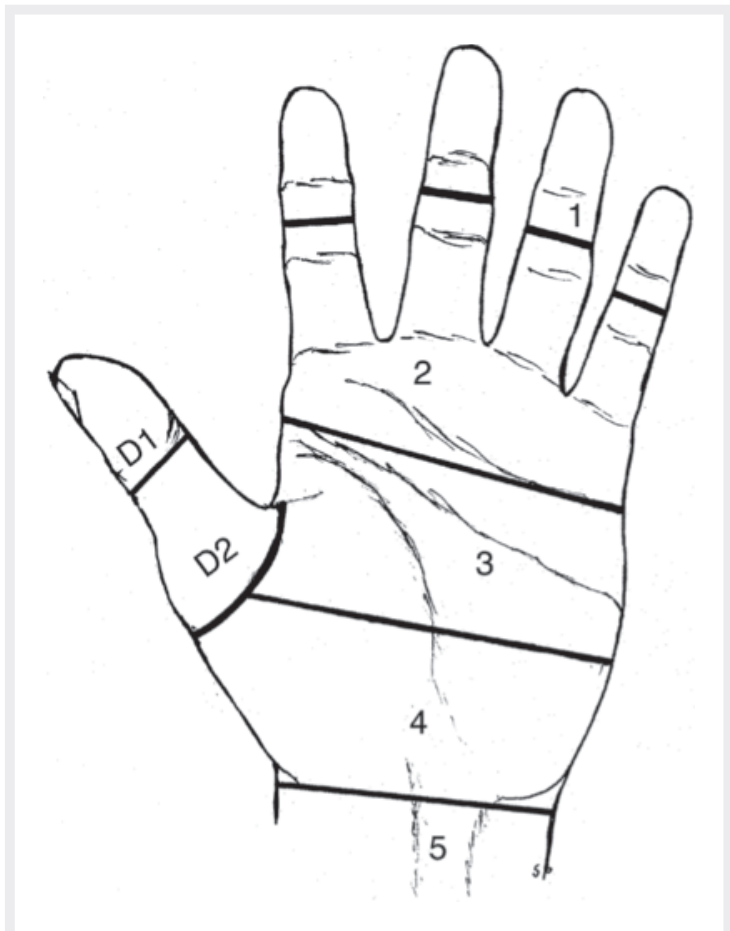

- Abb. 2 Zoneneinteilung bei Beugesehnenverletzungen der Hand. (Quelle: Hoffmann R, Krimmer H. Primäre Beugesehnennaht. In: Hoffmann R, Hrsg. Checkliste Handchirurgie. 4. Aufl. Stuttgart: Thieme; 2016)

das nicht der Fall, ist in der Regel eine primäre Verankerung der FDP-Sehne an den Knochen indiziert [5].

Zone 2 Die Behandlung von Beugesehnenverletzungen in Zone 2 ist chirurgisch gesehen eine besondere Herausforderung. Aufgrund der anatomisch engen Verhältnisse zwischen FDP- und FDS-Sehne sowie deren Sehnenscheide kann es hier schnell zur Entstehung von Sehnenadhäsionen kommen. Diese Verklebungen verhindern im Verlauf ein adäquates Sehnengleiten und erschweren somit die Nachbehandlung. Deshalb bedarf es hierbei einer speziellen chirurgischen Expertise. So kann es aufgrund der besonderen anatomischen Situation sinnvoll sein, auf die Naht einer der beiden Beugesehnen zu verzichten, um die Entstehung von Verklebungen durch zu starkes Sehnenreiben zu vermeiden [6].

Zonen 3-5 Verletzungen in diesen Zonen können in der Regel mit einer direkten Naht unproblematisch versorgt werden. Verklebungen sind hier seltener.

Zonen T1-T3 Die Zonen am Daumen werden durch den Buchstaben „T“ gekennzeichnet. Durchtrennungen in Zone $\mathrm{T} 1$ bedürfen häufig der Versorgung mittels LengemannAusziehnaht, da distal nicht mehr genügend Sehnenmaterial für eine Kernnaht vorhanden ist. Bei Verletzungen in den Zonen T2 und T3 kann eine 4-Strang-Naht der FPLSehne entsprechend des Vorgehens an den Langfingern erfolgen.

\section{Pathologie und Sehnenheilung}

Sehnengewebe besitzt einen deutlich niedrigeren Sauerstoffbedarf als andere Gewebearten. Diese tiefe metabolische Rate und ihre Fähigkeit, trotz niedrigem Sauerstoffangebot ausreichend Energie zu produzieren, sind essenziell, um Belastungen und Zugkräfte über lange Zeiträume auszuhalten. Nachteilig ist jedoch der daraus resultierende langsame Heilungsprozess nach einer Beugesehnendurchtrennung [7].

Heilungsphasen Die Sehnenheilung startet unmittelbar nach der Verletzung und wird in 3 unterschiedliche Phasen eingeteilt, die je nach Verletzungslokalisation und -art zeitlich variieren und sich überlappen können: Die Entzündungsphase dauert ca. 3-5 Tage. Aufgrund der zerstörten umliegenden Blutgefäße kommt es hierbei zur Ödembildung. Die genähte Sehne weist noch eine sehr geringe Belastbarkeit auf.

Die zweite Phase, auch Proliferationsphase genannt, dauert mehrere Wochen an. Das Areal um die Sehnennaht besteht nun aus unorganisiertem Granulationsgewebe. Etwa nach 10 Tagen weist die genähte Sehne die geringste Festigkeit auf [8]. Nun wird vermehrt Kollagen Typ III gebildet und auch bereits schrittweise in den optimalen Typ I umgewandelt. Somit steigt die Reißfestigkeit der Sehne ab diesem Zeitpunkt logarithmisch an.

In der 3. Phase, der Umbauphase, die über ein Jahr in Anspruch nehmen kann, kommt es zur Geweberestrukturierung. Die Kollagenfasern ordnen sich langsam wieder parallel zur Zugbeanspruchung an. Dieses Narbengewebe ist auf zellulärer Ebene allerdings weniger geordnet als normales Sehnengewebe. Die volle Reißfestigkeit einer unverletzten Sehne wird daher nie wieder erreicht [9][10].

Intrinsische und extrinsische Heilung Die Sehnenheilung kann währenddessen auf zwei unterschiedliche Weisen erfolgen. Es lassen sich hierbei intrinsische und 
extrinsische Heilungsprozesse unterscheiden. Zunächst kann die verletzte Sehne vom umliegenden Gewebe her repariert werden (=extrinsische Heilung). Blutgefäße sprossen von außen in das verletzte Sehnengewebe ein und unterstützen die Heilung. Hierbei entstehen allerdings auch Sehnenverklebungen von außen. Diese Adhäsionen erhöhen zum einen die Sehnenbelastbarkeit, verringern zum anderen jedoch auch deren Gleitfähigkeit. Hierdurch kann es langfristig zu einer schlechten Fingerbeweglichkeit, insbesondere einer eingeschränkten aktiven Beweglichkeit, kommen.

Bei intaktem Vinkulasystem und vorsichtiger operativer Präparation der Sehnenenden kann die Sehne allerdings auch vom Sehnengewebe selbst ausgehend heilen. Diese sogenannte intrinsische Heilung kann, im Gegensatz zur extrinsischen, ohne die Bildung von Adhäsionen ablaufen.

Nach Verletzung der Beugesehne bildet sich in jedem Fall Narbengewebe.

Ob eine verletzte Sehne vermehrt intrinsisch oder extrinsisch heilt, ist von vielen unterschiedlichen Faktoren abhängig und lässt sich somit nicht immer sicher beantworten. Zusammengefasst lässt sich jedoch sagen, dass eine vermehrt intrinsische Heilung bei geringerer Adhäsionsbildung eine bessere Beweglichkeit zur Folge hat. Jedoch ist eine so heilende Sehne aufgrund der fehlenden stabilisierenden Adhäsionen in der Nachbehandlung auch weniger belastbar [11][12][13].

Heilung ohne Operation Tritt die Sehnenheilung ohne vorherige Operation ein, besteht eine große Lücke zwischen den Sehnenstümpfen. Somit kommt es zu einer Vernarbung mit dem umliegenden Bindegewebe sowie zu einem minderwertigen Regenerat (geringere mechanische Stabilität und Reißfestigkeit) mit deutlich erhöhter Rerupturrate und schlechter Fingerfunktion [9][10]. Den Heilungsprozess der Sehne unterstützt man also mit einer adäquaten Adaption der beiden Sehnenenden am besten. Dies erreichen wir nur durch eine operative Versorgung.

\section{Diagnostik}

Für eine Operation ist eine adäquate Indikationsstellung notwendig. Hierfür bedarf es einer ausführlichen Anamnese und insbesondere einer genauen klinischen Untersuchung.

\section{Anamnese}

Zunächst erfragen wir den Unfallhergang. Der Traumamechanismus gibt bereits wichtige Informationen zur Einschätzung der Verletzungsart. Bei stumpfen Traumata kommt es eher zu Sehnenausrissen und Quetschverletzungen. Bei Schnittverletzungen ist eine glatte Durchtrennung der Sehnen zu erwarten. Diese Information ist für die Planung der operativen Versorgung wichtig. In diesem
Zusammenhang ist es auch entscheidend zu erfragen, ob sich die betroffenen Finger zum Zeitpunkt der Schnittverletzung in Beuge- oder Streckstellung befanden. Erfolgt die Schnittverletzung an einem Finger in Beugestellung, so ist der distale Sehnenstumpf weit distal der Schnittwunde, der zentrale Stumpf jedoch nur wenig proximal zu erwarten. In dieser Situation sind die Präparation und die operative Versorgung erschwert. Befindet sich der entsprechende Finger zum Zeitpunkt der Schnittverletzung in Streckstellung, so findet sich der distale Sehnenstumpf nahe der Wunde. Die Präparation ist in diesem Fall deutlich einfacher [4].

\section{Inspektion}

Im Ruhezustand zeigt die Hand einen zunehmenden Beugesehnentonus der Finger vom Zeigefinger hin zum Kleinfinger [14]. Jede Abweichung dieses Spannungsverlaufs ist Hinweis auf eine Beugesehnenverletzung. Bei isolierter Durchtrennung der FDP-Sehne an den Langfingern oder der FPL-Sehne am Daumen steht das Endgelenk in Streckstellung ( $>$ Abb. 3). Bei isolierter Durchtrennung der FDSSehne findet man einen insgesamt verminderten Fingerbeugetonus vor ( $\triangleright$ Abb.4). Bei komplettem Tonusverlust eines Fingers ist eine kombinierte Durchtrennung beider Beugesehnen anzunehmen. Der Finger befindet sich in einer auffälligen Streckhaltung ( $\triangleright$ Abb. 5).

\section{Klinische Untersuchung}

Da aufgrund der Nähe der Strukturen häufig Begleitverletzungen vorliegen, ist es grundsätzlich wichtig, die Sensibilität, Motorik und Durchblutung zu überprüfen. Hierbei ist die zügige Beurteilung der Durchblutung des Fingers distal der Schnittwunde entscheidend, da bei eingeschränkter Durchblutung eine sofortige operative Therapie mit Revaskularisierung, das heißt Wiederherstellung der Durchblutung, nötig ist. Die Durchblutung wird durch das Feststellen der Rekapillarisierungszeit im Vergleich mit den unverletzten Nachbarfingern erfasst.

Die aktive Funktionsprüfung der tiefen Beugesehne erfolgt bei auf der Tischebene festgehaltenem Mittelglied. In dieser Position fordert der behandelnde Arzt den Patienten auf, den jeweiligen Finger im Endgelenk zu beugen ( $\triangleright$ Abb.6a). Die aktive Untersuchung der oberflächlichen Beugesehne ist komplizierter. Beide Beugesehnen beugen über dem Mittelgelenk, sodass die Unterscheidung schwierig ist. Um eine aussagekräftige Testung durchführen zu können, muss also die FDP-Sehne funktionell ausgeschaltet werden. Anatomisch haben die tiefen Fingerbeugesehnen aller Langfinger einen gemeinsamen Muskelbauch. Sie bilden somit eine funktionelle Einheit. Wenn ein Finger weniger beweglich ist, sind es auch die drei anderen [5]. Also hält der Arzt den benachbarten Finger in Extension auf der Tischebene fest und fordert den Patienten auf, den zu untersuchenden Finger aktiv zu beugen. Die Flexion im Mittelgelenk kann dann nur durch eine intakte FDS-Sehne erfolgen ( $\triangleright$ Abb. $6 \mathbf{b}$ ). 


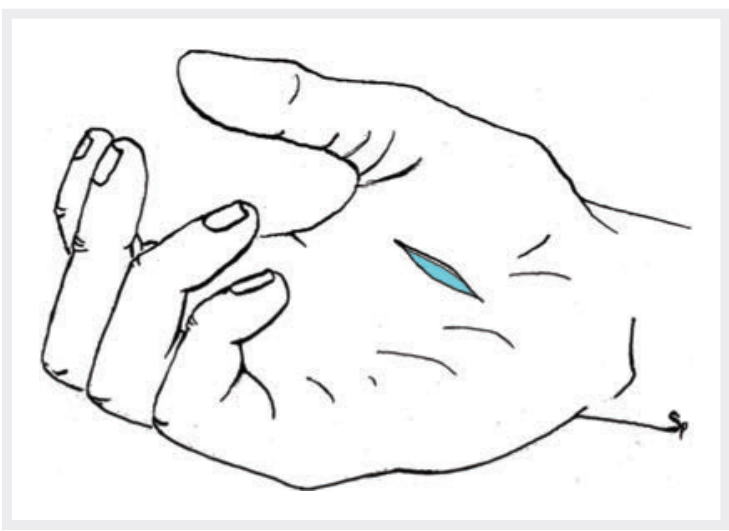

- Abb. 3 Isolierte Durchtrennung der FDP-Sehne III: Das Endgelenk befindet sich in Streckstellung. (Quelle: Hoffmann R, Krimmer H. Diagnostik. In: Hoffmann R, Hrsg. Checkliste Handchirurgie. 4. Auflage. Stuttgart: Thieme; 2016)

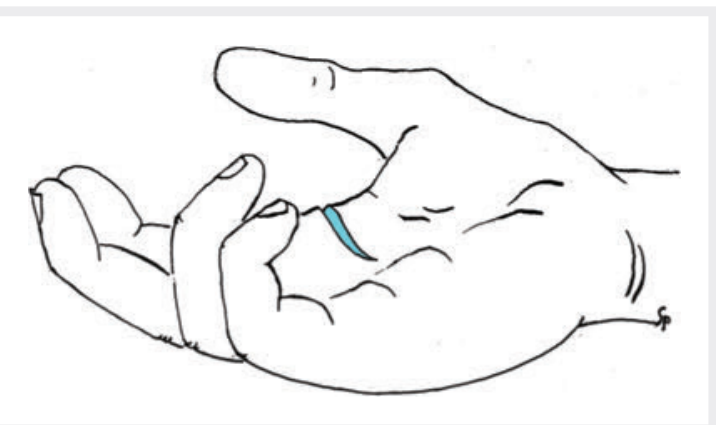

Abb. 4 Isolierte Durchtrennung der FDS-Sehnen II und III: Ein verminderter Beugetonus ist sichtbar. (Quelle: Hoffmann R, Krimmer H. Diagnostik. In: Hoffmann R, Hrsg. Checkliste Handchirurgie. 4. Auflage. Stuttgart: Thieme; 2016)

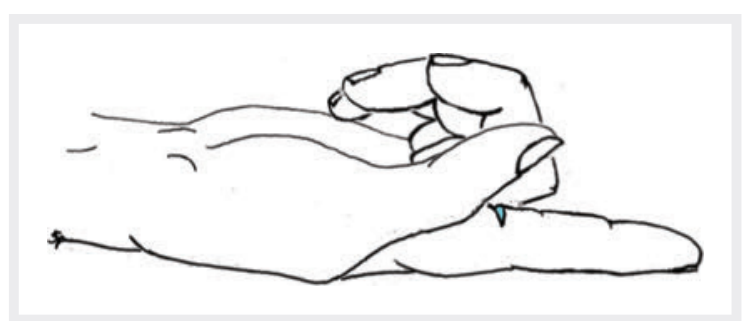

-Abb. 5 Kombinierte Durchtrennung beider Beugesehnen: Der Beugetonus ist aufgehoben. (Quelle: Hoffmann R, Krimmer H. Diagnostik. In: Hoffmann R, Hrsg. Checkliste Handchirurgie. 4. Auflage. Stuttgart: Thieme; 2016)

Bei fehlender Compliance des Patienten, Sprachbarriere oder bei Kleinkindern kann keine aussagekräftige Untersuchung der aktiven Fingerbeugung erfolgen. Hier kann der Tenodeseeffekt zur Beurteilung helfen [15]: Wenn eine Hand passiv im Handgelenk gestreckt wird, kommt es bei entspannter Haltung automatisch zu einer zunehmenden Fingerbeugung. Umgekehrt führt eine passive Beugung im

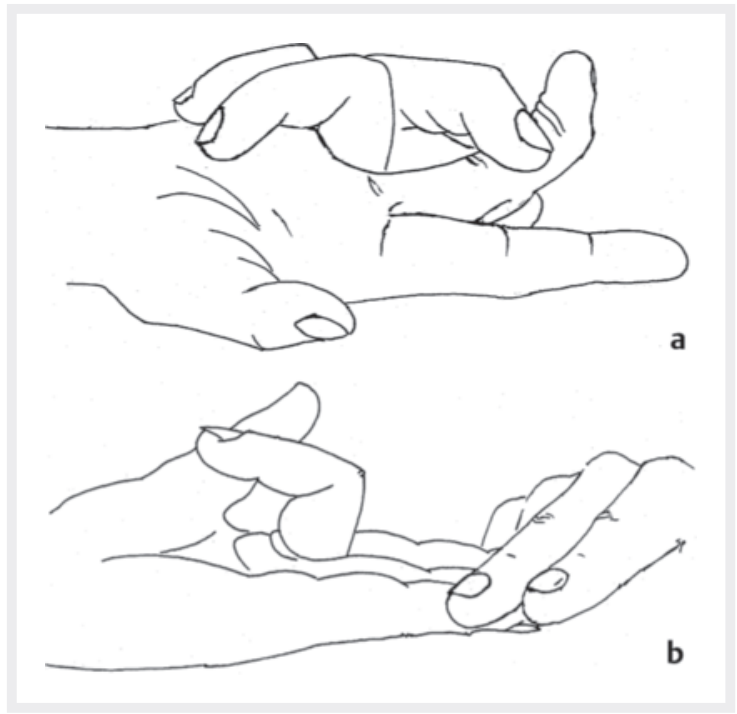

-Abb. 6 Klinische Funktionsprüfung der Beugesehnen: a) FDP-Sehne, b) FDS-Sehne. (Quelle: Hoffmann R, Krimmer H. Diagnostik. In: Hoffmann R, Hrsg. Checkliste Handchirurgie. 4. Auflage. Stuttgart: Thieme; 2016)

Handgelenk zu einer zunehmenden Fingerstreckung. Dieser Effekt lässt sich durch Druck auf die Muskulatur der entsprechenden Sehnen am Unterarm verstärken. Nach Beugesehnendurchtrennungen ist dieser Effekt verändert.

Wichtig ist zusätzlich immer eine Prüfung der Beugung gegen Widerstand. Wenn hierbei keine Beugung möglich ist oder diese schmerzhaft ist, kann der Arzt eine Teildurchtrennung der Beugesehne nicht ausschließen. Das bedeutet, dass er die Beugesehne operativ darstellen muss. Eine unversorgte Beugesehnenteilverletzung kann nämlich im Verlauf zu einer sekundären Sehnenruptur bei Belastung führen, wenn die Wunde nur mittels Hautnaht versorgt wurde.

\section{Röntgen}

Ergänzend kann ein Röntgenbild zum Ausschluss einer knöchernen Beteiligung oder des Vorliegens eines röntgendichten Fremdkörpers angefertigt werden.

\section{Beugesehnennaht}

Hinsichtlich einer optimalen Nahttechnik oder eines optimalen Nahtmaterials herrscht international bisher keine Einigung. Es ist sehr schwierig, die einzelnen relevanten publizierten Studien miteinander zu vergleichen, da unterschiedlichste Methoden zum Einsatz kamen. Dies führte auch zum Teil zu widersprüchlichen Ergebnissen. Allerdings gibt es einige Grundprinzipien, die beachtet werden sollten [16]. So steht fest, dass eine Erhöhung der Anzahl an Kernnähten (sog. Stränge) die Stabilität der Sehnennaht verstärkt, jedoch gleichzeitig die Gleitfähigkeit der Sehne durch das eingebrachte Fadenmaterial vermindert [17][18]. 


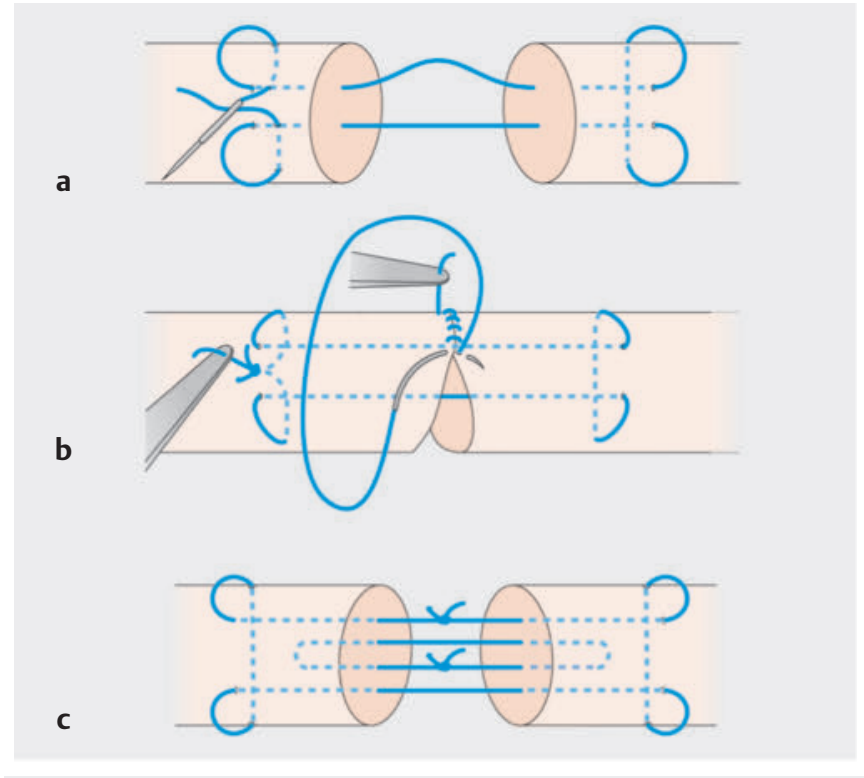

- Abb. 7 Beugesehnennähte. a) 2-Strang-Naht nach Kirchmayr-Kessler. b) Feinadaption. c) 4-Strang-Naht nach Strickland. (Quelle: Bühren V, Keel M, Marzi I et al. Beugesehnenverletzungen. In: Bühren V, Keel M, Marzi I, Hrsg. Checkliste Traumatologie. 7., komplett überarbeitete und erweiterte Auflage. Stuttgart: Thieme; 2011; grafische Umsetzung: Thieme Verlagsgruppe)

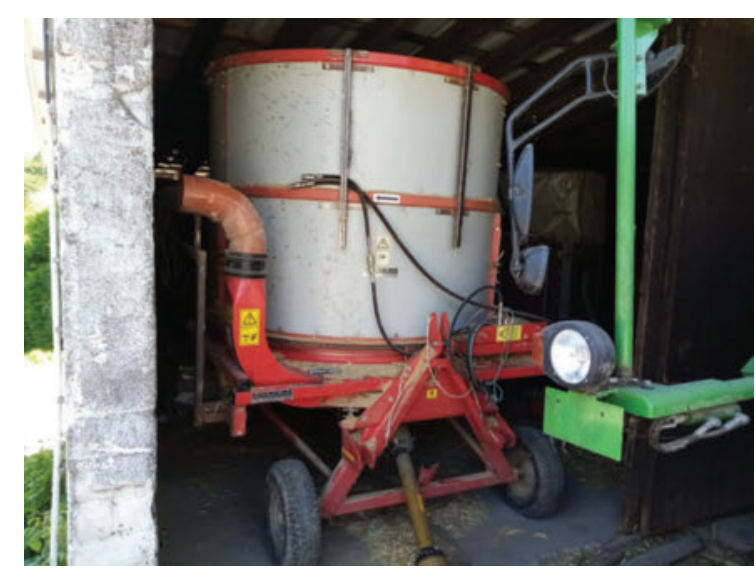

- Abb. 8 Beim Hantieren im Strohhäcksler durchtrennte sich Herr S. mehrere Beugesehnen. (Quelle: Privat)

-Abb. 7 zeigt häufig angewandte Techniken der Beugesehnennaht.

Unserer Meinung nach sind biomechanisch 4 Kernnähte die beste Wahl, um den Patienten in der Nachbehandlung frühzeitig aktiv beüben zu können und auf der anderen Seite nicht unnötig viel Fadenmaterial einzubringen. Deshalb wird von vielen Autoren derzeit die 4Strang-Naht als Standard angesehen, obwohl ein klinischer Vorteil gegenüber der einfacheren 2-Strang-Naht in Metaanalysen nicht gesichert ist [19].

Zu erwähnen gilt, dass andere Autoren beschreiben, dass auch nach 2-Strang-Naht eine aktive Beübung möglich sei. So fand sich bei aktiver Nachbehandlung kein statistisch signifikanter Unterschied bezüglich der Rupturrate bei Zweistrang- oder Mehrstrangtechniken [20]. Auch hinsichtlich des funktionellen Ergebnisses konnte in einzelnen Studien für Mehrstrangnähte keine Überlegenheit gezeigt werden [21].

Darüber hinaus muss sich der Chirurg entscheiden, wo er den Knoten platzieren soll. Eine Möglichkeit besteht darin, ihn zwischen die Sehnenstümpfe zu legen. Auch wenn dadurch eine gewisse Naht-Dehiszenz und eine Verminderung der Kontaktfläche der Sehnenstümpfe entstehen könnten, wurde in einer In-vivo-Studie keine Reduktion der Nahtstabilität festgestellt und sogar vermutet, dass diese Knotenlage einen positiven Anreiz auf die Sehnenheilung hat [22]. Eine zirkulär epitendinöse Feinadaptationsnaht dient zusätzlich dazu, minimale Lücken nach erfolgter Kernnaht zu schließen, um eine glatte Adaptation zu erreichen. Sie trägt außerdem bis zu 50 \% zur Gesamtstabilität bei [23].

\section{Fallbeispiel: Unfall auf dem Bauernhof}

Herr S. erlitt im Rahmen seiner landwirtschaftlichen Tätigkeit auf dem Bauernhof eine Durchtrennung der Beugesehnen von Zeige-, Mittel- und Ringfinger der linken Hand. Er ist Linkshänder und von Beruf Landwirt. Ihm war anfangs noch nicht bewusst, dass aufgrund dieser Verletzung seine gesamte berufliche Zukunft und die Existenz seines Bauernhofes auf dem Spiel standen. Bei seiner notfallmäßigen Vorstellung in der Klinik berichtete er, dass er eine Verstopfung im Strohhäcksler ( $\mathbf{A b b} \mathbf{b}$. 8) lösen wollte. Hierfür habe er den Auswurf abgeschraubt und in die Maschine gefasst. Obwohl er zuvor daran gedacht hatte, die Stromzufuhr zu unterbrechen, lief die Strohmühle noch nach. Hierbei verletzte er seine linke Hand an der rotierenden Klinge.

In der klinischen Untersuchung zeigten sich Durchblutung und Sensibilität intakt. Inspektorisch fiel mir sofort ein kompletter Tonusverlust der Finger der linken Hand auf. Passend zur Höhe der Schnittverletzung in Zone 2, proximal, hatte ich den Verdacht auf eine Durchtrennung der FDP- und FDS-Sehnen II-V. Dieser erhärtete sich durch die Funktionsprüfung. Der Bauer konnte Zeige- bis Kleinfinger nicht mehr aktiv beugen. Radiologisch bestanden keine Auffälligkeiten. Wir stellten die Indikation zur sofortigen operativen Versorgung und führten eine 4-StrangNaht mit Feinadaptation der FDP-Sehnen II-IV sowie U-Naht der FDS-Sehnen II-IV durch. A Abb. 9 zeigt die Hand nach der operativen Versorgung.

Die Nachbehandlung erfolgte für 6 Wochen nach dem modifizierten Kleinert-Schema. Nach 12 Wochen konnte er die Arbeit auf seinem Bauernhof wiederaufnehmen. Seine Existenz konnte somit durch die moderne Beugesehnenbehandlung in Zusammenarbeit von Chirurg und Ergotherapeut gesichert werden. 


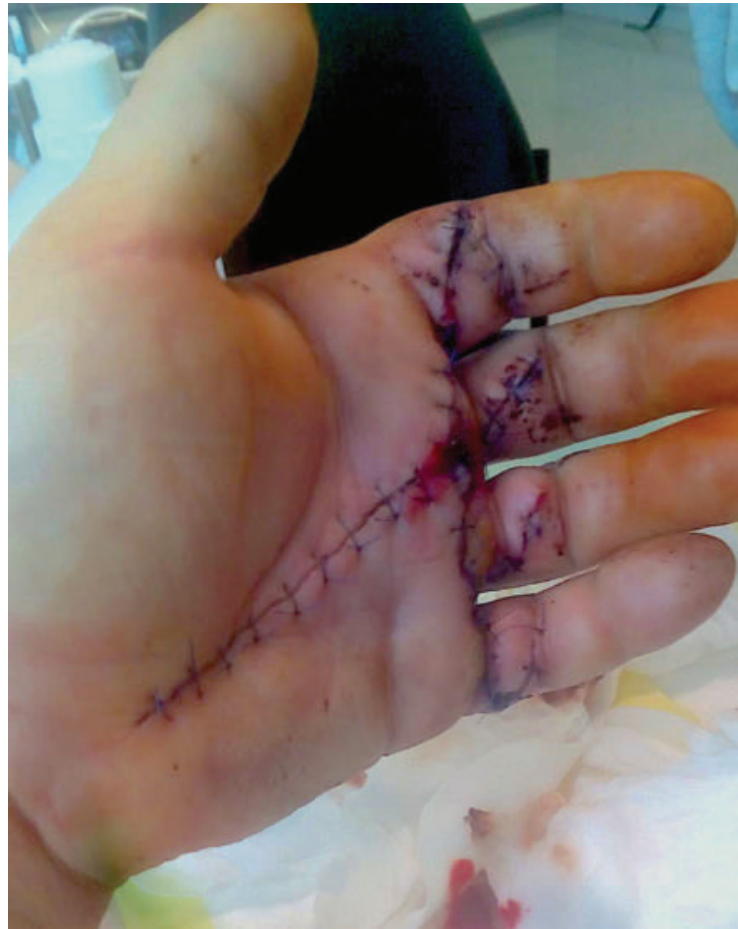

Abb. 9 Die Hand von Herrn S. nach Schnittverletzung und operativer Versorgung. (Quelle: Privat)

\section{Autorinnen / Autoren}

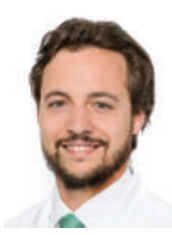

\section{Dr. med. Wolfram A. Heitzmann}

Assistenzarzt der Plastischen und Handchirurgie, studierte 2007-2014 Humanmedizin an der Julius-Maximilians-Universität Würzburg, war 2015-2016 als Assistenzarzt in der Klinik für Handchirurgie Bad Neustadt an der Saale tätig und arbeitet seitdem in der Plastischen Chirurgie in Köln-Merheim.

\section{Korrespondenzadresse}

Krankenhaus Köln-Merheim

Abteilung für Plastische Chirurgie

Ostmerheimer Str. 200

51109 Köln

Deutschland

\section{Literatur}

[1] Bundesverband für ambulantes Operieren e. V. Handverletzungen Nummer eins bei Arbeitsunfällen. Im Internet: https://www.operieren.de/e3224/e10/e1705/e1707/e1708; Stand: 18.7.2020

[2] Ederer IA, Nusche A, Daigeler A, Bösch C. Versorgung von frischen Beugesehnenverletzungen. Trauma und Berufskrankheit 2018; 20: 171-176
[3] Schmidt HM, Lanz U. Chirurgische Anatomie der Hand. 2., Überarbeitete u. aktualisierte Aufl. Stuttgart: Thieme; 2003

[4] Hoffmann R. Checkliste Handchirurgie. Stuttgart: Thieme; 2009

[5] Schreuders TA. The quadriga phenomenon: A review and clinical relevance. J Hand Surg Eur Vol 2012; 37: 513-522

[6] Kotwal PP, Ansari MT. Zone 2 flexor tendon injuries: Venturing into the no man's land. Indian J Orthop 2012; 46: 608-615

[7] O'Brien M. Structure and metabolism of tendons. Scand J Med Sci Sports 1997; 7(2): 55-61

[8] Preissner P. Beugesehnenverletzungen (31.12.2019). Im Internet: www. handchirurgie.org/pdf/Sehnen/Beugesehnen.pdf; Stand: 11.08 .2020

[9] Frankewycz B. Untersuchung zweier bioresorbierbarer Matrizen (PGA und Kollagen I) auf ihr tenogenes Regenerationspotential unter Einfluss zweier unterschiedlich vorbehandelter mesenchymaler Stammzellen (BMSC und MSC) beim Totaldefekt der Achillessehne der Ratte [Dissertation]. München: Ludwig-Maximilians-Universität; 2012

[10] Seradge $\mathrm{H}$. Elongation of the repair configuration following flexor tendon repair. J Hand Surg Am 1983; 8(2): 182-185

[11] Mason ML, Allen HS. The rate of healing of tendons - an experimental study of tensile strength. Annals of Surgery 1941; 113: 424-459

[12] Potenza AD. Critical evaluation of flexor tendon healing and adhesion formation within artificial digital sheaths. An experimental study. J Bone Joint Surg 1963; 45A: 1217-1233

[13] Strickland JW. The scientific basis for advances in flexor tendon surgery. J Hand Ther 2005; 18: 94-110

[14] Hahn P, Unglaub F, Spies CK. Diagnostik der Beugesehnenverletzungen der Hand. Orthopäde 2015; 44: 743-747

[15] Langer M, Surke C, Wieskötter B. Prinzipien der Sehnenbehandlung: Beugesehnen. In: Towfigh $\mathrm{H}$, Hierner R, Langer M, Friedel R, (Hrsg). Handchirurgie. 2. Aufl. Berlin: Springer; 2011

[16] Pillukat T, Schoonhoven J van. Suture techniques and materials in flexor tendon surgery. Trauma und Berufskrankheit 2016; 18: 264-269

[17] Cao Y, Tang JB. Biomechanical evaluation of a four-strand modification of the Tang method of tendon repair. J Hand Surg Br 2005; 30: 374-378

[18] Thurman RT, Trumble TE, Hanel DP et al. Two-, four-, and sixstrand zone II flexor tendon repairs: An in situ biomechanical comparison using a cadaver model. J Hand Surg Am 1998; 23: 261-265

[19] Savage R. In vitro studies of a new method of flexor tendon repair. J Hand Surg Br 1985; 10: 135-141

[20] IFSSH Scientific Committee on Flexor Tendon Repair: Trends in flexor tendon surgery over the last 10 years. IFSSH 2015. Im Internet: https://www.ifssh.info/pdf/Fle xor_tendon_Repair_2015.pdf Zugegriffen: 18.7.2020

[21] Hardwicke JT, Tan JJ, Foster MA, Titley OG. A systematic review of 2-strand versus multistrand core suture techniques and functional outcome after digital flexor tendon repair. J Hand Surg Am 2014; 39: 686-695

[22] Kubota $\mathrm{H}$, Aoki M, Pruitt $\mathrm{DL}$ et al. The tensile strength of various peripheral circumferential repair techniques in canine flexor tendons. J Orthop Sci 1996; 1: 136-139

[23] Kamal RN, Yao J. Evidence-based medicine: Surgical management of flexor tendon lacerations. Plast Reconstr Surg. 2017; 140(1): 130e-139e 